\title{
Doppler analysis of superior mesenteric artery blood flow in preterm infants
}

\author{
E LEIDIG
}

Department of Neonatology, University Children's Hospital, Tübingen, Federal Republic of Germany

SUMmary Transcutaneous Doppler ultrasound measurements were made of the superior mesenteric artery of 22 preterm infants (mean birth weight $600-2580 \mathrm{~g}$ ) to investigate the response of intestinal blood flow to feeding. Analysis of the data in relation to milk intake showed a significant change in peak velocity and mean velocity; peak velocity before feeding increased from a mean baseline value of $34 \mathrm{~cm} / \mathrm{s}$ in fasting infants to $46 \mathrm{~cm} / \mathrm{s}$ when less than $50 \mathrm{~g}$ milk/kg body weight a day were given, and up to $56 \mathrm{~cm} / \mathrm{s}$ when $50 \mathrm{~g}$ or more of milk/kg body weight a day were given. The corresponding values for mean velocity were 10,15 , and $22 \mathrm{~cm} / \mathrm{s}$, respectively. The pulsatility index decreased from 0.94 to 0.90 and 0.86 as milk volumes were increased. This investigation showed characteristic changes in the response of intestinal blood flow to feeding: 15 minute peak velocity and mean velocity rose significantly 15,45 , and 90 minutes after feeding, whereas the pulsatility index fell. Flow rate peaked 45 minutes after feeding.

Several methods have been used to study blood flow of human intestine. ${ }^{1}$ Only the transcutaneous Doppler flow method, however, has become a routine procedure for the evaluation of intestinal circulation. Recently there have been single reports of the possibilities of duplex scan examinations of the superior mesenteric artery in adults. ${ }^{23}$ and a Doppler study of superior mesenteric artery blood flow measurements in healthy newborns has been carried out. ${ }^{4}$ With the exception of a short abstract, ${ }^{5}$ I know of no other investigation on intestinal circulation in preterm or infants born at full term.

The duplex scan technique (real time scanner combined with pulsed Doppler equipment) allows precise localisation of a defined volume within the superior mesenteric artery from which the Doppler flow signal is sampled. This study describes the method of measuring blood flow in the superior mesenteric arteries of premature infants. 'Normal' values and characteristic changes in flow patterns of 22 preterm neonates were correlated with enteral feeding and the amount of milk given; peak velocity, mean velocity (integral of the maximum velocities over cardiac cycle), and pulsatility index were analysed.

\section{Patients and methods}

The data obtained from 22 preterm infants (gesta- tional age range $25-36$ weeks, weight at examination range $600-2850 \mathrm{~g}$, and postnatal age range 1 hour30 days) were evaluated. At ultrasound examination the general condition of the infants was good; oxygen and carbon dioxide tensions and blood pressures were normal, and their abdomens were free of gas. There were no signs or symptoms of necrotising enterocolitis. Eight patients with hyaline membrane disease required mechanical ventilation. Doppler investigations were carried out in these infants during the first days of life only, to minimise the influence of severe patent ductus arteriosus. Because the infants tolerated the procedure well, sedatives were unnecessary. All preterm infants were fed predefined, gradually increasing, quantities of milk through a gastric tube at intervals of 2 , $2 \cdot 5$, or 3 hours - that is, 12,10 , or eight feeds a day. The milk was injected by syringe into the feeding tube over a one to five minute period.

An ATL Ultramark 8 duplex scanner (Scientific Medical Systems), consisting of a real time $7.5 \mathrm{MHz}$ sector scanner and associated $5 \mathrm{MHz}$ pulsed Doppler flowmeter, was used. The signals were analysed using an integral fast Fourier transform processor. The superior mesenteric artery was localised by real time imaging on a horizontal or longitudinal abdominal section, which allowed placement of the sample volume (axial length $1 \cdot 5-3 \mathrm{~mm}$, lateral dimension $2 \mathrm{~mm}$ ) at the desired point within the 
superior mesenteric artery-that is, about 2 to $4 \mathrm{~mm}$ distal from where it branches off from the descending aorta (fig 1). In most cases the angle between ultrasound beam and blood flow in the vessel was close to 0 degrees $\left(\right.$ range, $0-20^{\circ}$ ); angle correction of the velocities was made automatically. Because of the low inspiratory peak pressures used in the ventilated infants, respiratory movements did not interfere with the measurements. The diaphragmatic movements were comparable with those in normal respiration. The position of the sample volume was optimised by listening to and observing the spectral display, then the recordings were made (usually in the longitudinal plane). The spectrally analysed flow signals of the Doppler shift (digitised every $5 \mathrm{~ms}$ ) were recorded in real time on a thermoprinter with time on the horizontal axis, Doppler shift frequencies on the vertical axis (velocity), and a grey scale indicating the quantity of blood flowing at that particular velocity (fig 2). After recording the optimised Doppler signals for 10 to 20 seconds, five homogeneous cardiac cycles were measured. Investigations were carried out in fasting infants, preprandially, and in conjunction with milk intake $(15,45$, and 90 minutes after feeding). The influence of the quantity of milk was verified in the following three groups set up according to daily milk intake: group A-fasting preterms, group B-<50 ml milk/ $\mathrm{kg}$ body weight a day, and group $\mathrm{C}-50 \mathrm{ml}$ or more of milk/kg body weight a day. Statistical analysis was by the Kruskal-Wallis and the Wilcoxon rank sum tests. A $p$ value of $<0.05$ was taken as significant.

\section{Results}

$$
\text { Peried Wolien reads. Forty velurdia }
$$

Sixteen of the 22 preterm infants were examined once, five twice, and one three times with varying quantities of food. A total of 29 Doppler recordings were made for the three groups. Within each group (A, B, or C) each baby had Doppler measurements made once.

Individual fasting or before feeding values for peak velocity, mean velocity, and pulsatility index in the three groups are shown in fig 3 , and the mean (SD) result for each group is shown in the table. Peak velocity and mean velocity increased as the quantity of milk increased; the pulsatility index decreased analogously.

The changes of the flow measurements after feeding are shown in fig 4 . The data are based on 60 Doppler recordings of 16 preterm infants for whom, in addition to before feeding basal values, had 15 and 45 minute $(n=4)$ or 15,45 , and 90 minute $(n=12)$ values also recorded after feeding. Each curve represents a different patient. These recordings include patients with varying food intake (groups B and C), and therefore mean values and

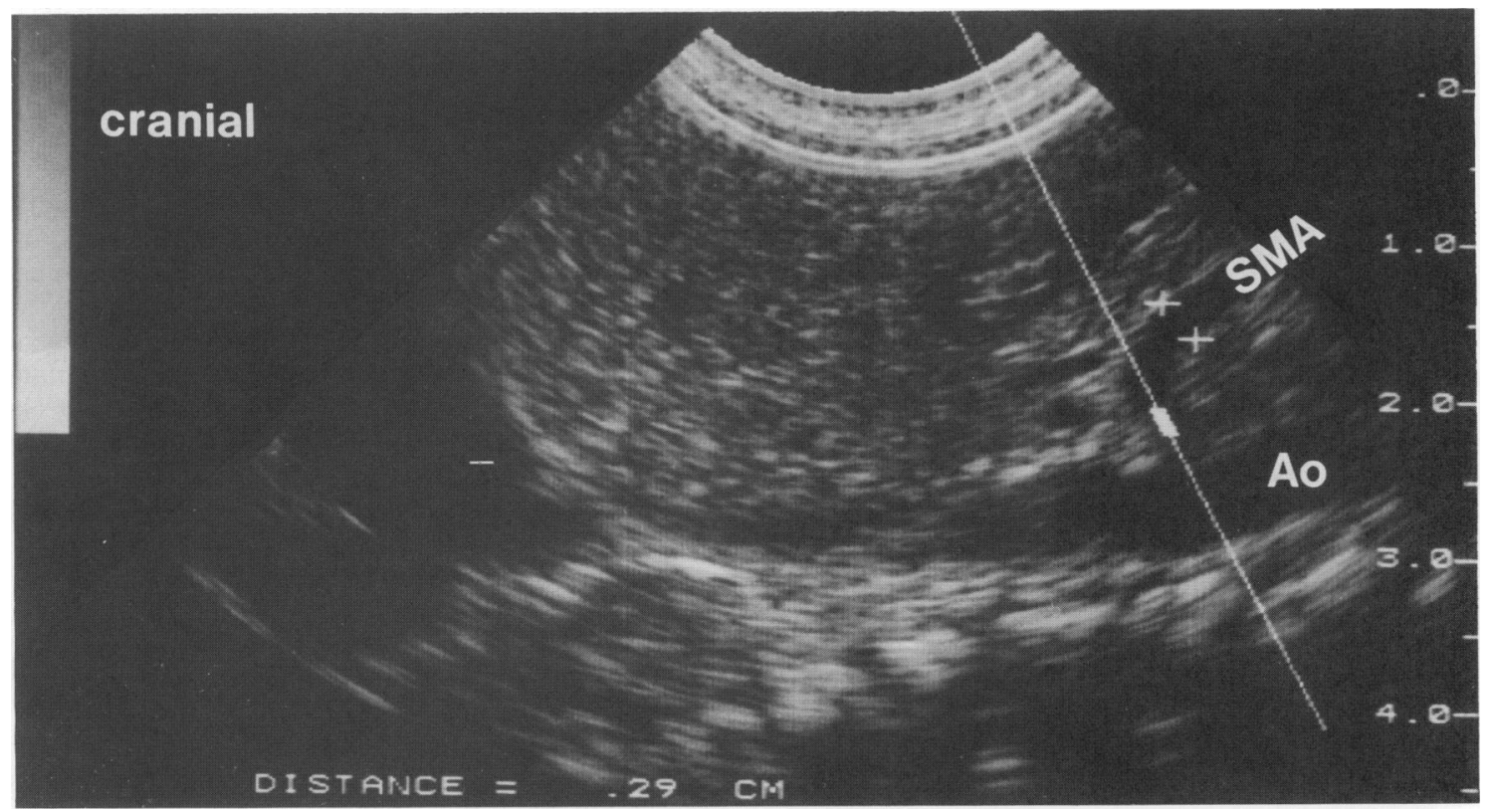

Fig 1 Sample volume within superior mesenteric artery (SMA). Diameter is $2.9 \mathrm{~mm}$ (marked by $X)$. Ao=Aorta. 


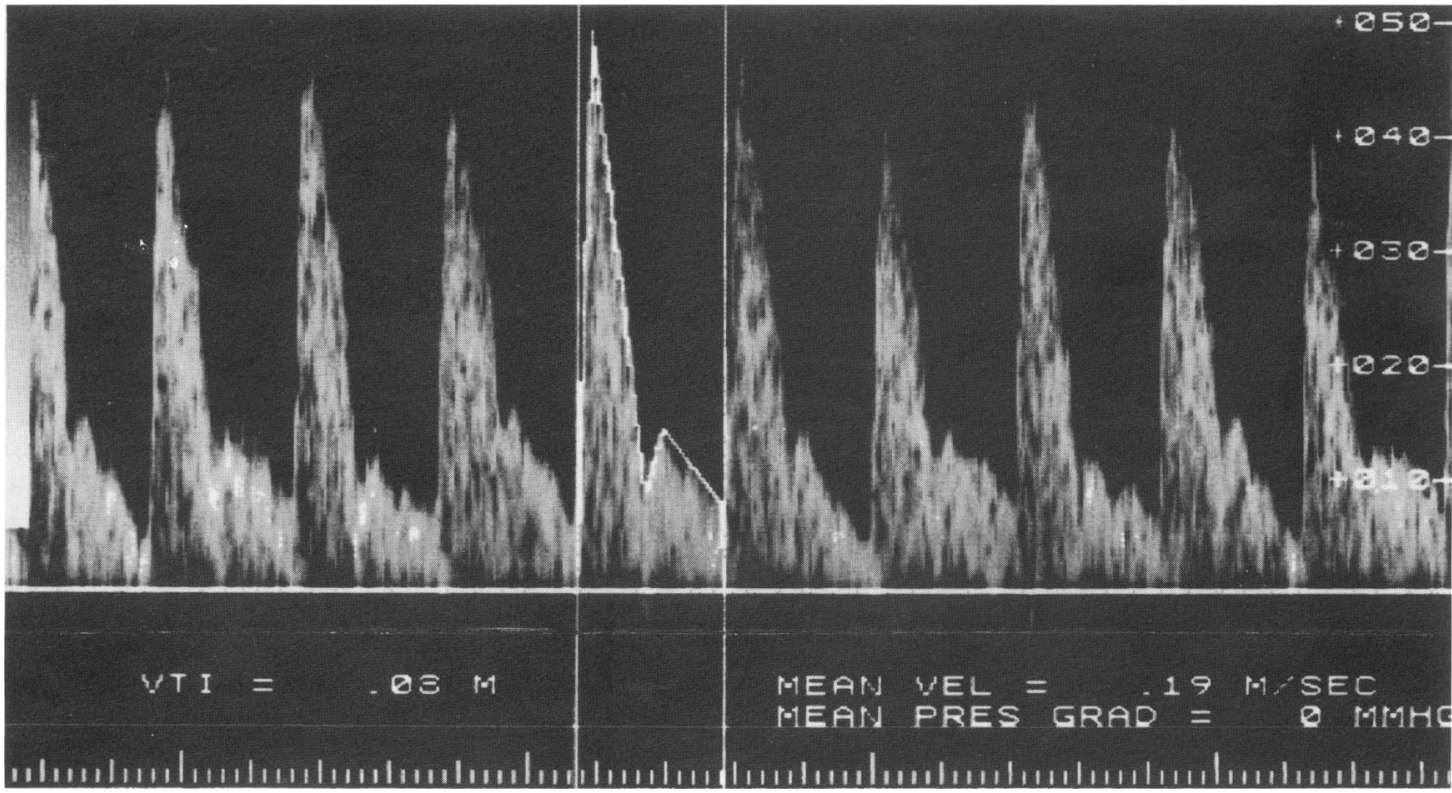

Fig 2 Flow signal of superior mesenteric artery. Preprandial velocity is shown on the vertical axis $(40-45 \mathrm{~cm} / \mathrm{s})$. Area below white outline represents mean velocity (integral of maximum velocities over cardiac cycle), which is $19 \mathrm{~cm} / \mathrm{s}$.

standard deviations after feeding were not calculated because their wide distributions would have been misleading. The postprandial increase of flow rate was analysed by the Wilcoxon rank sum test. The curves show a steep, significant rise in peak velocity and mean velocity 15 minutes after feeding, whereas pulsatility index fell. Peak velocity rose significantly again between the 15 and 45 minute values. The flow rate peaked at 45 minutes; the minimum interval between feedings was two hours.

\section{Discussion}

Blood flow disturbances are an integral part of the aetiology of neonatal abdominal disorders, especially necrotising enterocolitis. ${ }^{6-8}$ Measurements of intestinal circulation in adults ${ }^{2}$ by pulsed Doppler ultrasound of the superior mesenteric artery showed

Table Mean (SD) Doppler parameters in the three groups

\begin{tabular}{llll}
\hline & $\begin{array}{l}\text { Group } A \\
(n=7)\end{array}$ & $\begin{array}{l}\text { Group } B \\
(n=12)\end{array}$ & $\begin{array}{l}\text { Group } C \\
(n=10)\end{array}$ \\
\hline Peak velocity $(\mathrm{cm} / \mathrm{sec})$ & $34(6)$ & $46(10)$ & $56(6)$ \\
Mean velocity (cm/sec) & $10(2)$ & $15(4)$ & $22(5)$ \\
Pulsatility index & $0.94(0.06)$ & $0.90(0.09)$ & $0.86(0.09)$ \\
\hline
\end{tabular}

significant changes in flow measurements in association with food intake. Qamar et al ${ }^{9}$ rcported that the response of superior mesenteric artery blood flow even differed depending on the caloric content of the meal (carbohydrate, fat, protein).

Improvement of sonographic techniques with high resolution scan heads $(7.5 \mathrm{MHz})$ has made imaging of minute vessels like the superior mesenteric artery in newborns and very low birthweight infants possible. The combined Doppler method has considerably widened our knowledge of neonatal intestinal circulation. Leidig ${ }^{4}$ investigated flow patterns and normal values in healthy newborns. The present study shows that preterm infants are able to regulate blood flow in the superior mesenteric artery in association with milk intake; in addition, baseline values are reported.

Evaluating the correlation between intestinal perfusion and postpartum age is difficult, because our patients received milk orally 12 to 24 hours after birth. The loading effect of milk on the intestine, therefore, cannot be differentiated from possible changes resulting from increasing postnatal age. The pure time dependent effect can be shown only after several days of fasting. Given that with the regular daily increase in milk intake the postnatal age is associated with the quantity of milk, testing the effect of varying quantities of milk also indirectly 
examined the time dependency of blood flow in the superior mesenteric artery. Postnatal ages in group $\mathrm{A}, \mathrm{B}$, and $\mathrm{C}$ were as follows: group $\mathrm{A}-1-18$ hours, group $B-1-9$ days, and group $C-10-30$ days.

The significant increase in peak velocity and mean velocity and the declining pulsatility index in fig 3 shows that the quantity of milk serves as an index of superior mesenteric artery perfusion. In contrast to Daneman et $\mathrm{al}^{5}$ (who found no significant consistent decrease in pulsatility index after enteral feeding in six premature infants) the preterm infants examined and analysed in this study, showed a definite change in all flow measurements after meals-that is, decreasing pulsatility index, increasing peak velocity, and increasing mean velocity (fig 4). Because the pulsatility of the recorded Doppler wave form is associated with the vascular impedance downstream to the point of measurement, ${ }^{10}$ intestinal vascular resistance after feeding reaches its nadir between 15 and 45 minutes after feeding.

The pulsatility in Doppler recordings, especially in premature infants receiving assisted ventilation, depends primarily on the severity of patent ductus arteriosus. Mean flow velocity values are lower because of the reduced diastolic flow, which in some cases is even retrograde with pulsatility index values of more than $1 \cdot 00$. The three pulsatility index values over 1.00 in fig 3 were measured in ventilated infants and should be attributed to the effect of a mild patent ductus arteriosus. With the exception of these raised pulsatility index values, the flow measurements did not differ between ventilated infants and those with spontaneous respiration (critically ill infants with severe patent ductus arteriosus were excluded). A reduction of intestinal circulation during feeding is to be expected in critically ill ventilated babies, particularly those with demonstrable holodiastolic backflow into the superior mesenteric artery. In such cases, a delay in enteral feeding and gradual increase of food intake (maximum daily increase, $5 \mathrm{~g}$ food/ $\mathrm{kg}$ body weight) are indicated to prevent such gastrointestinal complications as extensive bowel gas, vomiting, and malnutrition. Further investigations are necessary to establish the efficacy of individual superior mesenteric artery flow measurements for the evaluation
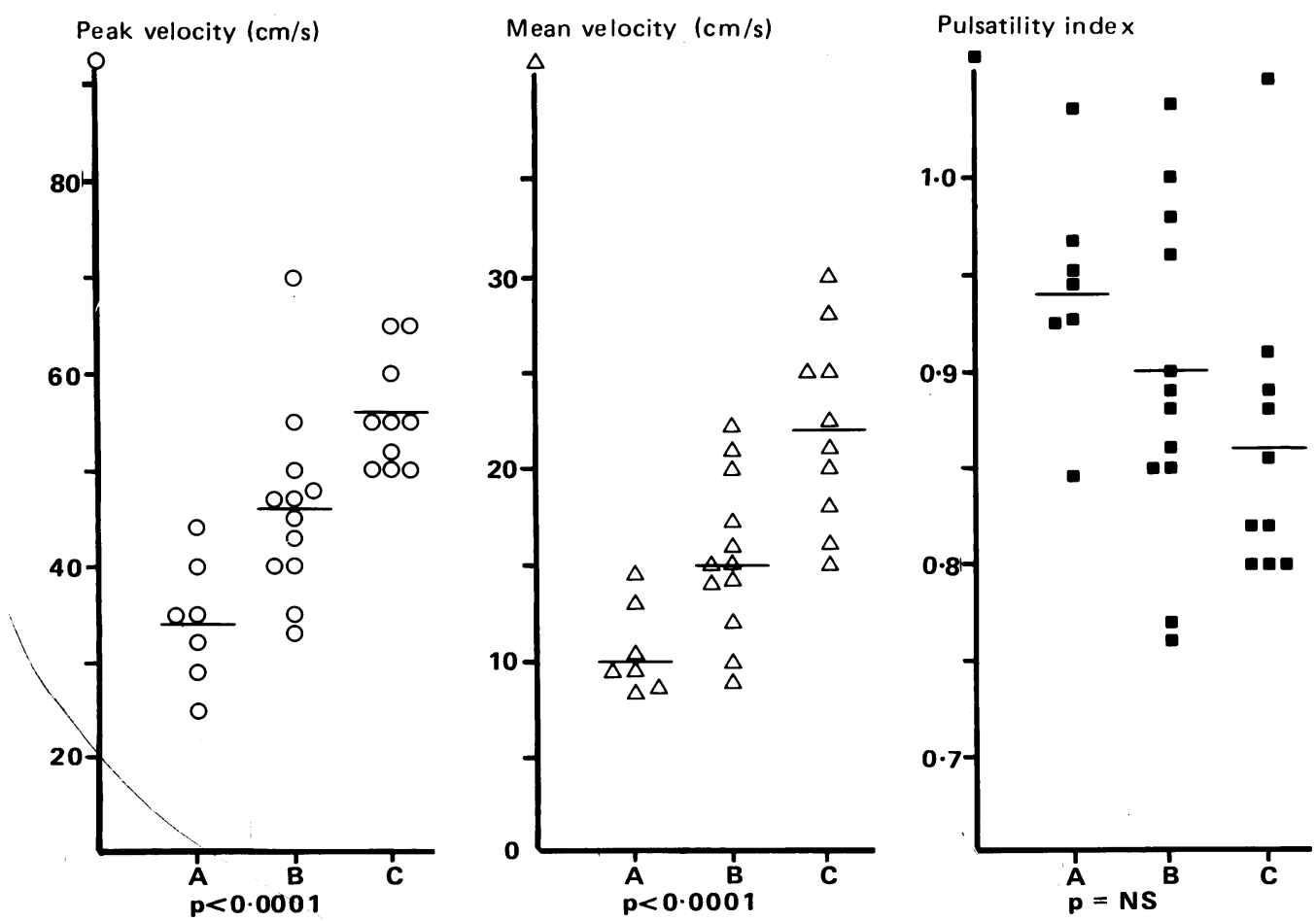

Fig 3 Peak velocity, mean velocity, and pulsatility index in babies who had not been fed $(A)$ or who had received $<50 \mathrm{ml} \mathrm{milk} / \mathrm{kg}$ body weight $(B)$ or $\geqslant 50 \mathrm{ml} \mathrm{milk/kg}$ body weight $(C)$. Measurements in groups $B$ and $C$ were made immediately before a feed. Horizontal lines indicate mean of individual values. 

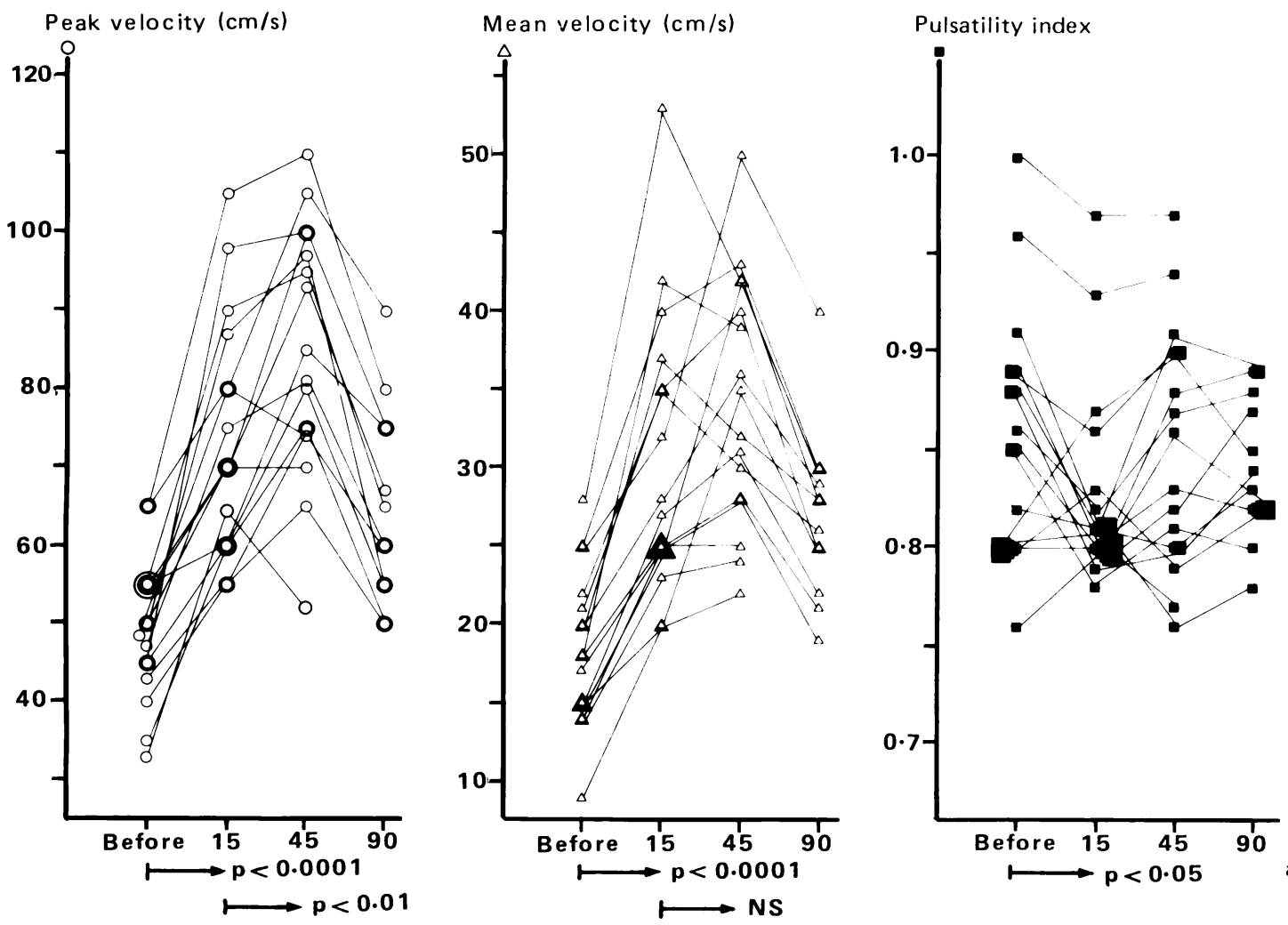

Fig 4 Response of peak velocity, mean velocity, and pulsatility index to feeding $(n=16)$. Large symbols represent identical values.

and quantification of reduced intestinal perfusion in newborns and premature infants with gastrointestinal disease.

\section{References}

1 Tepperman BL, Jacobsson ED. Measurement of gastrointestinal blood flow. Ann Rev Physiol 1982;44:71-82.

2 Taylor KJW, Burns PN, Woodcock JP, Wells PNT. Blood flow in deep abdominal and pelvic vessels: ultrasonic pulsed-Doppler analysis. Radiology 1985; 154:487-93.

3 Qamar MI, Read AE, Skidmore R, Evans JM, Wells PNT. Transcutaneous Doppler ultrasound measurement of superior mesenteric artery blood flow in man. Gut 1986;27:100-5.

${ }^{4}$ Leidig E. Pulsed Doppler ultrasound blood flow measurements in the superior mesenteric artery of the newborn. Pediatr Radiol 1988 (in press).

5 Daneman A, Babyn P, Garcia P, Stringer DA, Hellman J. Doppler sonography of intraabdominal vessels in premature infants. Pediatr Radiol 1987;17:342 (A).
${ }^{6}$ Obladen M. Necrotizing enterocolitis. Monatsschr Kinderheilkd 1986;134:515-9.

${ }^{7}$ Hackett GA, Campbell S, Gamsu H, Cohen-Overbeek T, Pearce JMF. Doppler studies in the growth retarded fetus and prediction of neonatal necrotizing entercolitis, hacmorrhage, and neonatal morbidity. $\mathrm{Br}$ Med $J$ 1987;294:13-6.

${ }^{8}$ Decg KH, Gerstner R, Bundscherer F, Harai G, Singer H, Gutheil H. Doppler sonographic detection of reduced flow velocities in the truncus coeliacus in infants with patent ductus arteriosus Botalli in comparison to a healthy control group. Monatsschr Kinderheilkd 1987;135:24-9.

9 Qamar MI, Read AE, Mountford R, Skidmore R, Wells PNT. Effect of carbohydrate, fat and protein on superior mesenteric artery blood flow in man. Gut 1984;25:1154(A).

${ }^{10}$ Evans DH, Barrie WW, Asher MJ, Bentley S, Bell PR. The relationship between ultrasonic pulsatility index and proximal arterial stenosis in a canine model. Circ Res 1980;46:470-5.

Correspondence to Dr Eberhard Leidig, Department of Neonatology, University Children's Hospital, Rümelinstrasse 23, D-7400 Tübingen, Federal Republic of Germany.

Accepted 29 June 1988 